\title{
Rape as loss of honor in the discourse of Moroccan rape trials
}

\section{Fatima-Zahra Lamrani}

The invited talk by Fatima-Zahra Lamrani has been published as:

Lamrani, Fatima-Zahra (2013). Rape as loss of honor in the discourse of Moroccan rape trials. Language and Law 2: 1-18. DOI: http://dx.doi.org/10.14762/j11.2013.n1 